\title{
INDEX OF ISSUE 2
}

A

Abaclat Tribunal, 108

Amendments to the Arbitration and Conciliation Act 1996 (246th Report), 180

American Express, 217

Anationalists, 155

Andhra Pradesh High Court, 168

Arbitral legal order, 139-158 is semi-autonomous, 140 as semi-autonomous social fields, $146-157$

Arbitration, 146

idea of, 149

raison d'être of, 146

Arbitration Act, 1940 (1940 Act), 169, 173, 244

Arbitration (Protocol and Convention) Act, 1937, 169, 170, 173

Arbitration agreement, 152, 153

existence or validity of, 216

non-arbitrability, 216-218

scope, termination or waiver of, 215

violation of public policy, 216-218

Arbitration and Conciliation Act, 1996 (1996 Act), 173-179

Arbitration and Conciliation Act, 1996 (Arbitration Act), 234

Arbitration and Conciliation (Amendment) Act, 2015 (2015 Amendment), 174

Arbitration and Conciliation (Amendment) Act, 2019 (2019 Amendment), 187

Arbitration and Conciliation (Amendment) Bill, 2015, 185

Arbitration and Conciliation (Amendment) Ordinance, 2015, 185

Arbitration-mediation (Arb-Med), 122, 127-128

Arbitration-mediation-arbitration (Arb-MedArb), 117, 122, 128-131

Arb-Med-Arb Protocol (AMA Protocol), 130
Article 3(1) and (2) of the Singapore

Convention, 121

Article III of the New York Convention, 121

Article II(3) of the New York Convention, 120

Article 1(e) of the Geneva Convention, 170

Article 5(1) of the Singapore Convention on Mediation, 131

Article 5(1)(a) of the Singapore Convention on Mediation, 134

Article 5(2) of the Singapore Convention on Mediation, 132

Article V(1)(a) and (e) of the New York Convention, 120

Article V of the New York Convention, 120

Article V(1)(a) of the New York Convention, 134,152

Article V(2) of the New York Convention, 120,132

Article $\mathrm{V}(2)(\mathrm{b})$ of the New York Convention, 170

Asia, enforcement mechanism vs. drawing lessons, 113-137

Asian International Arbitration Centre (AIAC), 124

Associate Builders v. Delhi Development Authority, 182, 184, 185

B

Bahraini Free Arbitration Zone, 157

Banks, 93-112

appointed trustee of Qatar Trust, 99

as claimants, 95-101

hedging agreement, 104

loans, 104

qualifies as an investor, 95, 111

treaty-based international investment arbitration, 94

Bansi Dhar \& Ors v. Ajudhia Prasad \& Ors, 165 
Barbados International Trusts Act, 100

Barbados law, 100

Barbados-Venezuela BIT, 99, 100

Beijing Arbitration Commission (BAC), 124

Belize, arbitration against, 105

Berger, Klaus, 144

Bermann, George, 136, 141, 155

BG Group, 206

Bharat Aluminium Co v. Kaiser Aluminium Technical Services, Inc (BALCO), 174

Bhatia International v. Bulk Trading SA \& Anr, 174

Bilateral investment treaty (BIT), 94

Blue Bank, 98-101

Blue Bank International \& Trust (Barbados) Ltd. v. Venezuela, 95, 98-101, 111

Bombay High Court, 170, 179, 186

Born, Gary, 199, 216

British Caribbean Bank, 105, 109

British Caribbean Bank Ltd v. Belize, 104, 108, 109

'Broad' view, 171

C

Case law, 133, 224

Central Bank of Sri Lanka, 106, 110, 111

Central Inland Water Transport Corpn $\mathcal{E}$ Anr v. Brojo Nath Ganguly \& Anr, 167

Centrotrade Minerals \& Metals Inc v. Hindustan Copper Ltd, 178

Ceylon Petroleum Corporation (CPC), 106

Chilling effect, 128

China International Economic and Trade Arbitration Commission (CIETAC), 124

Choice of law analysis, 195, 198-204, 208-209

Commercial arbitration, 94

Commission's 246th Report, 181

Common law, 163-164

Common Law V. Civil Law Jurisdictions, 196-197

Constitution of India, 167

Contra bonos mores, 164

Contract Act, 164, 176, 179, 184, 240, See also Indian Contract Act, 1872

Contracting Party, 96

Contract law, 164

Convention on International Settlement Agreements Resulting from Mediation, See Singapore Convention on Mediation
Convention on the Recognition and Enforcement of Foreign Arbitral Awards, See New York Convention

Court interference, limits of, 183

Court of Appeal, 133

D

Dallah Real Estate, 152

Dallah Real Estate v. Government of Pakistan, 152, 153

Delhi Development Authority v. RS Sharma, 178

Delhi High Court, 161, 237, 241

Delhi Transport Corporation v. DTC Mazdoor Congress \& Ors, 167

Denning, Lord, 162

Deutsche Bank, 106, 108

Deutsche Bank AG v. Sri Lanka, 106-108, 110

Dispositif, 197, 218

Domestic laws, 146

Dugard, John, 144

E

Ehrlich, Eugene, 141, 154

Enderby Town Football Club Ltd v. Football Assn $L t d, 162$

Energy Charter Treaty, 107

Enforceability, 129

Enforcement, mode of, 121-122

English arbitral laws, 152

English Arbitration Act of 1996, 148, 152

English Case, 152

English law, 153

Equalization of effects, doctrine of, 208

Essential Commodities Act, 1955, 242

Event of Default, 96

Execution of Foreign Arbitral Awards, 147

Ex turpi causa, 243

\section{F}

Factual Matrix, 236-237

Federation of Oil Seeds and Fats Association (FOSFA), 236

Fischer-Lescano, Andreas, 144

Foreign Awards Act, 171, 173, 179, 237, 240, 244

Foreign Awards (Recognition and Enforcement) Act, 1961, 170, 234

Foreign Exchange Management Act, 1999, 190

Foreign Exchange Regulation Act, 1973, 173

French law, 152, 153

French private international law, 153 
Fundamental policy of Indian law, 173, 180-185, 191, 242

G

Gaillard, Emanuel, 149, 150, 151

Galanter, Marc, 143-144

Gao Haiyan v. Keeneye Holdings Ltd, 126, 127

Geneva Convention on Execution of Foreign Arbitral Awards, 169

Genevan Convention of 1927, 147

Geneva Protocol of 1923, 147

Geneva Protocol on Arbitration Clauses, 169

German-Sri Lanka BIT, 106, 110

Gherulal Parakh v. Mahadeodas Maiya \& Ors, 166

Global Pound Conference Survey, 114

Goode, Roy, 144-145

Gouvernement du Pakistan - Ministere des Affaires Religieuses $v$. Dallah Real Estate and Tourism Holding Co, 153

Greece, jurisdiction of Tribunal, 102

Griffiths, John, 142-143

Gurmukh Singh v. Amar Singh, 169

\section{$\mathrm{H}$}

Hague Conference, 147

'Hands-off' approach, 234

Hard law, 194

Harmonization, process of, 147

Hedging Agreement, 106, 107, 110

High Court of Singapore, 162

High Courts, 173, 187

Hindustan Zinc Ltd v. Friends Coal Carbonisation, 178

Hong Kong Court of Appeal, 126

Hong Kong Court of First Instance, 126

Hong Kong International Arbitration Centre (HKIAC), 124

Host State, 94, 98, 101, 107, 108, 112

Hub for international arbitration, 188

Hub of arbitration, 163

Hybrid approach, 201-202

Hybrid mechanisms, 122-123

I

ICCA's Guide to the Interpretation of the 1958 New York Convention: A Handbook for Judges, 136

ILA Recommendations, 202-204, 218

Independent Power Tanzania Limited (IPTL), 95 loan agreements with Malaysian banks, 95-98

India

basis of precedent, 164

development of public policy, 163-188

Foreign Awards Act, 170-171

international commercial arbitration, 188

1940 Act dealt with domestic arbitrations, 169

public policy doctrine, 160, 162-164, 166, 169, 188

signed New York Convention, 169-170

Indian arbitration, 159-192

Indian Arbitration Act, 1940, 235

Indian arbitration law, 162

Indian Contract Act, 1872, 164-169, 238

Indian domestic arbitration, 173

Indian High Courts, 165

Indian Institute of Arbitration and Mediation (IIAM), 124

Indian law, 188, 235

fundamental policy of, 173, 180-185, 242

Indian Penal Code, 161

Indian-seated international commercial arbitration, 188, 189

Interests of India, 180, 184, 186

International arbitration, 140, 198

res judicata in, 197-206

theoretical framework, 140-157

International Arbitration Act, 133

International Business Community (IBC), 145

International business community (societas mercatorum), 144-146

International Centre for Dispute Resolution (ICDR), 124

International Chamber of Commerce (ICC), 124, 145

International Chamber of Commerce's International Court of Arbitration, 170

International Commercial Contracts, 154

International commercial law, 143, 148

International Law Association (ILA), 199

International Mediation Institute, 115

International Standards Approach, 202-204

International trade law, 144

Invent new public policy, 168

Investment arbitrations, 93-112, 94

themes, 111

Investments, 94

concept of, 101

definition of, 101-107 
German-Sri Lanka BIT, 106-107

location of, 107-108

Slovakia-Greece BIT, 102-103

UK-Belize BIT, 105-106

Investment treaties, 108-109

substantive protections, 108-111

Investors, 94

concept of, 95

ISDA Master Agreement, 93-94

\section{$\mathrm{J}$}

JG Engineers Pvt Ltd v. Union of India $\mathcal{E}$ Anr, 178 Judicial approach, 185

Jurisdictional court decisions, 211-218

Jurispersuasion, 141

Justice, 184, 186

K

Kompetenz-kompetenz, doctrine of, 204, 210, 215, 219

\section{L}

Law Commission of India, 180, 184

Law Commission's 246th Report and Supplement, 185

Law Commission's 246th Report and Western Geco, 180-184

Law, definition of, 142-143

Law of Treaties, 96, 103, 105

Laws of Barbados, 98

Lewis v. Circuit City Stores, 205

Lex arbitri, 150, 156, 200, 201

Lex mercatoria, 144, 151-152

Loan agreements, 105

benefit of, 108

Loan and Security Agreements, 105, 106, 108, 109

Loans, 104

London Court of International Arbitration (LCIA), 124, 155

M

Mainland Chinese institutions, 129

Malaysian banks, 95, 97

Malinowski, Bronisław, 141-142

McDermott International Inc v. Burn Standard Co $L t d, 178$

Mediation-arbitration (Med-Arb), 122-127

Mediation Institute of the Stockholm Chamber of Commerce (SCC), 124
Model Law on Arbitration, 134

Modi, Narendra, 188

Moore, Sally, 142-143, 154

Morality, 184, 186

Multilateral investment treaty, 94

Murlidhar Aggarwal \& Anr v. State of Uttar Pradesh \& Anr, 166, 167

Mustill, Lord, 145

Mutadis mutandis, 207

$\mathrm{N}$

NAFED v. Alimenta, 233-244

'Narrow' view, 171, 185

National Agricultural Co-operative Marketing Federation of India v. Alimenta $S A$, 190, 240, 242-244, See also NAFED v. Alimenta

National Association of Securities Dealers (NASD), 205-206

National laws, 199-204

Navtej Singh Johar \& Ors v. Union of India, 161, 162

Nawab Salar Jung III, 168

Naz Foundation v. Govt of NCT of Delhi $\mathcal{E}$ Ors, 161

New Delhi International Arbitration Centre (NDIAC), 188

New York Convention, 94, 115

on arbitration, 113-137

and Geneva Convention awards, 173

pro-enforcement bias, 191

and Singapore Convention on Mediation, 113-117

Singapore Convention on Mediation for lessons, 131-137

travaux préparatoires of, 125

1996 Act

amendments to, $187-188$

with conciliation, 173-174

with domestic arbitration, 173-174

enforcement of foreign awards, 173-174

public policy of India, 175

supplementary provisions, 173-174

$\mathrm{O}$

Oil and Natural Gas Corpn Ltd v. Saw Pipes Ltd, 176-179, 181

ONGC Ltd v. Western Geco International Ltd, 181-186

Ong Ming Johnson v. Attorney-General, 162 
Oudh Judicial Commissioner's Court in 1924, 165

\section{$\mathbf{P}$}

Pakistan, government of, 152, 153

Pandora's box, 236

Paris Cour d'Appel, 153

Party autonomy, 146

Patent illegality, 176, 177, 184, 189, 192

Paulsson, Jan, 149-151, 153-155, 157

Penal Code (Singapore), 162

Permanent Court of Arbitration, 147

Permanent Court of International Justice, 195

Phulchand Exports Ltd v. OOO Patriot, 178, 179

Pluralism, 154

Pollock, Frederick, 144

Porgiton v. Spain, 107

Poštová banka, a.s. v. Greece, 101, 105

Power purchase agreement (PPA), 95

Prima-facie, 212

Private international law, 147, 149-150

Pro-arbitration, 157, 173, 219

Pro-arbitration regime, 214

Pro-arbitration scheme, 207

Pro-enforcement approach, 189

Pro-enforcement bias, 234

Proviso, 181

Public international law, 144

Public policy, 159-163

development of, 163-188

domestic and international arbitration awards, 191-192

of foreign arbitral awards, 170-171

heads of, 164

impeached, 239-240

interpretation of, 165

limiting scope of, 188-192

limits of court interference, 183-184

narrow prongs of, 169-173

of private international arbitration, 172

shifting sands of, 159-192

violation of, 216-218

Public policy of India, 171, 175, 176, 178, 179, $182,186,237,240$

Public policy test, 162, 183, 189, 190, 192

Putrabali, 153

\section{Q}

Qatar Trust, 99, 100 substantial investor, 99

Queen Mary International Arbitration Survey, 94
$\mathrm{R}$

Ramaswamy, J. K., 167

Ratanachand Hirachand v. Askar Nawaz Jung $\mathcal{E}$ Ors, 168

Ratione materiae, 101, 102, 104, 107

Ratione personae, 95, 100

Rattan Chand Hira Chand v. Askar Nawaz Jung $\mathcal{E}$ Ors, 168, 169

Regulation of States, 147

The Renusagar Era, 169-173

Renusagar Power Co. Ltd. v. General Electric Co, 170, 173, 176, 177, 179-181, 185, 234, 235,240

Renvoi, 153

Res judicata choice of law analysis, 198-204, 208-209 court decision effects in international arbitration, 206-218

court or arbitrator to decide, 204-206

doctrine of, 194-197

ILA Recommendations, 202-204

in international arbitration, 197-206

jurisdictional court decisions, 211-218

national laws, 199-202

prior court decisions, 207-208

requirements of, 198-204, 209-211

scope of doctrine, 196-197

Roman maxims, 198

S

Sajjid Yar Jung, 168

Santi-Romano, 154

Saudi Arabian company, 152

Schaffstein, Silja, 214

Section 34

of the Arbitration and Conciliation Act, 1996, 173-179

challenges to domestic award, 175, 177, 178,181

public policy of India, 176, 178, 179, 182, 183

Semi-autonomous, 139-158

Semi-autonomous social field (SASF), 142

arbitral legal order as, 146-157

definition of, 148

international business community (societas mercatorum), 144-146

role in international business community, 143-144

Shri Lal Mahal Ltd v. Progetto Grano SpA, 179, 180

SIAC-SIMC Arb-Med-Arb service, 130 
Singapore Convention on Mediation defences to enforcement, 119-121

enforcement as arbitral award, 122-123

Arb-Med, 127-128

Arb-Med-Arb, 128-131

Med-Arb, 123-127

lessons from New York Convention, 131-137

mode of enforcement, 121-122

New York Convention and, 113-117

procedural requirements, 117-119

Singapore International Arbitration Centre (SIAC), 130

Singapore International Dispute Resolution Academy, 122

Singapore International Mediation Centre (SIMC), 130

Slovak bank, 101

Slovakia-Greece BIT, 101, 102-104

Societas mercatorum, 144, 145, 149

Soft laws, 146, 194

Sri Lanka, territorial nexus with, 107-108

Ssangyong Engg \& Construction Co Ltd $v$. National Highways Authority of India, 185-187, 189, 192, 235

Standard Chartered Bank, 95, 97, 98, 111

Standard Chartered Bank Hong Kong, 95, 97

Standard Chartered Bank v. Tanzania, 95-98, 111

Standard Chartered Bank v. Venezuela, 99

Standard Chartered Sherwood, 97

Stare decisis, doctrine of, 198

State-centric perspective, 151

State sovereignty, theory of, 149-150

Strong, Stacie, 115

Supranational legal order, 153

Supreme Court, 171, 173, 179, 183, 185

cases

Associate Builders, 182

Bhatia, 174

Brojo Nath Ganguly, 167

Gurmukh Singh, 169

Phulchand, 178

Rattan Chand Hira Chand, 168

Renusagar, 170

Saw Pipes, 176

demonstrate fundamental policy of Indian law test, 189-190

of India, 161, 234

of Sri Lanka, 106, 110

Suresh Kumar Koushal v. Naz Foundation, 161

Sutlej Construction Ltd v. State (UT of

Chandigarh), 185

Swiss company, 237
$\mathrm{T}$

Tanzanian Loans, 98

Teubner, Gunter, 144

Third Restatement, 200, 203, 205

Toth, Orsolya, 144-145

Travaux préparatoires, 125

Tribunal, 94-97, 99-101, 103

expropriation of loans, 109

fair and equitable treatment standard, 110

loans and bonds, 104

Trust Deed, 100

246th Report, Supplementary Report to, 184

2015 Amendment and the Ssangyong Clarification, 185-188

U

UK-Belize BIT, 105, 106, 108

UK-Tanzania BIT, 95-98

Ultra vires, 167

UNCITRAL Guide to the New York Convention, 136

UNCITRAL Model Law, on International Commercial Arbitration, 136-137, 173

UNDIROIT Principles, 154

UNIDROIT, 145

Union of India v. Col LSN Murthy, 178

Union of India v. Recon, 186

United Nations Commission on International Trade Law (UNCITRAL) Model Law on International Commercial Arbitration, 1985, 173

United Nations Commission on International Trade Law (UNCITRAL) Working Group II, 116

United Nation's Convention on the Recognition and Enforcement of Foreign Arbitral Awards (New York Convention), 169

United Nations Economic and Social Council (ECOSOC), 148

US federal Securities Act, 217

US Supreme Court, 205, 206

$\mathrm{V}$

Venture Global Engg LLC v. Tech Mahindra Ltd, 185

Vienna Convention, 96, 103, 105

Vietnam International Arbitration Centre (VIAC), 124

Vijay Karia $\mathcal{E}$ Ors v. Prysmian Cavi E Sistemi SRL \& Ors, 189, 241-244 
W

Wednesbury principle, 182, 184, 186, 189 Wilberforce, Lord, 148-149

Wilko, 217

Working Group, 118, 120, 121, 132-135
World Sport Group (Mauritius) Ltd v. MSM Satellite (Singapore) Pte Ltd, 179

$X$

Xian Arbitration Commission, 126 
